\title{
EXAMINING THE QUALITY OF KNOWLEDGE TRANSFERS - THE DRAFT OF AN EMPIRICAL RESEARCH
}

\author{
Grum, Marcus (1); \\ Klippert, Monika (2); \\ Albers, Albert (2); \\ Gronau, Norbert (1); \\ Thim, Christof (1) \\ 1: University of Potsdam; \\ 2: Karlsruhe Institute of Technology (KIT)
}

\begin{abstract}
Already successfully used products or designs, past projects or our own experiences can be the basis for the development of new products. As reference products or existing knowledge, it is reused in the development process and across generations of products. Since further, products are developed in cooperation, the development of new product generations is characterized by knowledge-intensive processes in which information and knowledge are exchanged between different kinds of knowledge carriers. The particular knowledge transfer here describes the identification of knowledge, its transmission from the knowledge carrier to the knowledge receiver, and its application by the knowledge receiver, which includes embodied knowledge of physical products. Initial empirical findings of the quantitative effects regarding the speed of knowledge transfers already have been examined. However, the factors influencing the quality of knowledge transfer to increase the efficiency and effectiveness of knowledge transfer in product development have not yet been examined empirically. Therefore, this paper prepares an experimental setting for the empirical investigation of the quality of knowledge transfers.
\end{abstract}

Keywords: Knowledge management, New product development, Evaluation

\section{Contact:}

Klippert, Monika

Karlsruhe Institute of Technology (KIT)

IPEK Institute of Product Engineering

Germany

monika.klippert@kit.edu 


\section{INTRODUCTION}

Product development can be described as a knowledge-intensive process. This process relies on the competence of its participants, which have to handle complex tasks and cope with the transfer of sticky knowledge (Grum et al., 2019). The challenges arising from the relationship between competency, complexity and stickiness need to be addressed during process design in order to use organization's resources optimally and address their influence on duration, cost and quality of the product development activities: First, experiments about knowledge-intensive work have shown, that the characterization of knowledge transfers has a quantitative effect on necessary duration of a successful transfer (Gronau and Grum, 2019). Second, costs are incurred due to faulty and lengthy transfer processes or the potential of the knowledge resource remains untapped (Drucker, 1994). Third, the potential that knowledge transfers have an effect on the quality of process outcomes is topic of anecdotal evidence but has not been assessed thus far. This paper therefore outlines a two-year research project, which investigates the situations and conditions of knowledge transfers their effect on outcome quality. It thus answers the following main research question: How can a practical experimental environment be designed that allows the quantitative investigation of factors influencing the quality of knowledge transfer outcomes?

This paper rather outlines the corner points of this research project than presenting a ready to go description of concrete experiments. An experiment realization and data collection will be conducted thereafter. Thus, section two presents a theoretical foundation. It presents the product development context and ties it to knowledge transfers, which is further elaborated using existing studies on the impacting factors and quality assessment. Knowledge-intensive artifacts are introduced and defined as the pivotal element for the conjunction of knowledge transfers and quality-based outcomes. The third section elaborates the design requirements for an experimentation of knowledge transfers and hypothesizes influencing factors. The fourth section outlines design components, which allow to transfer experimentation results among situations and generalize correctly. The fifth and sixth section demonstrate and evaluate the draft of an empirical study and the transfer of study results. Finally, a conclusion points to the concrete next steps for an implementation and appraises the progress critically.

\section{THEORETICAL FOUNDATION AND UNDERLYING CONCEPTS}

\subsection{Product Development Context}

In many cases, the quality of product development processes determines the competitiveness of industrial companies. Therefore, the survival of companies in dynamic environments depends more than ever on their ability to use existing knowledge, generate new knowledge and exploit their innovative and creative potential (Sommerlatte et al., 2006). The model of PGE - Product Generation Engineering describes the phenomenon that products are developed in generations (Albers et al., 2015) whereby existing tacit knowledge, experiences and the explication of former development outcomes are reused. For economic and risk-analytical reasons, the aim is to achieve the desired functions and properties of the new product with the least possible modifications (Eckert et al., 2010).

Since iterative, complex and insecure processes of knowledge creation and transfer are the basis of product development (Lindemann et al., 2008), a coupled view on business process (BPM) and knowledge management (KM) are ideal (Albers et al., 2014). Aiming for the investigation of by the KM induced modifications of knowledge transfers makes knowledge-intensive processes of PGE the ideal research context, because process outcomes can be evaluated in regard with the time required and the quality achieved.

\subsection{Knowledge Transfers}

Knowledge transfers are considered as the process of three stages: the identification of knowledge, its transmission from knowledge carrier to knowledge receiver, and its utilization by the knowledge receiver (Gronau and Grum, 2019). Particularly, the utilization is essential, so that knowledge transfer can be observed through its results or manifestations.

In order to specify the experimentation of knowledge transfers and to capture the dynamic nature of the transfer process, models used in process-oriented KM have proven to be helpful (Gronau and Grum, 2019). By analyzing the resulting models, knowledge changes can be identified during the knowledge 
transfer and by whom they occur. Moreover, the conditions of knowledge transfers can be managed efficiently (Albers et al., 2019b).

The basic constructs in modeling are the different forms of tacit and explicit knowledge (Nonaka and Takeuchi, 1995) and embodied knowledge (Gronau, 2019) as well as their conversion from one form to another. Since the activities of knowledge creation, transmission and application employ these conversions, at this research, the temporal description of a knowledge-intensive process (process perspective) will be separated from the iterative activity description of knowledge creation, transfer and application (knowledge perspective). So, each task, knowledge transfer and conversion can be specified individually. Since these will be embedded in the experiment process, the coarse of time of transfers can be captured and visually represented. The transfer becomes controllable and one point for interventions (Grum and Gronau, 2018). The output of activities (knowledge-intensive artifacts) are the observable signposts in these knowledge-intensive processes.

\subsection{Quality Measurement}

In general, quality can either be defined as an absence of deficiencies or as characteristics of a product or service that are necessary to fulfill the desired needs (ASQ, 2020). However, quality is a subjective category, that captures the output of specific actions, which can be assessed retrospectively as conformance with predefined requirements and specifications (Crosby, 1979). For this research, this means that characteristics of process outcomes to fulfill certain needs must be defined before the knowledge transfers are realized. Measuring points directly need to evaluate inhowfar the requirements on desirable characteristics are fulfilled. According to ISO (2019) from software development context, measures can quantify quality by counting the fulfilled requirements or calculating the degree of partial fulfillment, if a scale is defined beforehand. In regard with knowledge-intensive process outcomes, the following two kinds of measurements can be established.

Since only explicit outputs in form of artifacts can be assessed, tacit knowledge outcomes need to be put in action and transformed into artifacts in order to measure their quality. Regrettably, there are gaps when putting tacit knowledge into action, which arises from barriers such as the inability to perform the action, the institutional environment or the individual motivation (Connelly et al., 2014). Thus, only a portion of the acquired tacit knowledge will result in an observable output. For example, if a person reads a paper on mechanics, understanding it and constructing a machine are different things. Indirect measurements therefore need to consider theses barriers in addition either through subjective self-assessment (motivation, ability) or by describing the conditions and situations (trust, time-pressure, IT-support, competition, incentives) under which the transfer took place and including the hampering factors in the assessment (Rhodes et al., 2008).

Explicit outputs on the other hand are either documented (explicit knowledge) or physically present (embodied knowledge). The quality of these can be assessed either structural (logical structure, completeness) or with regard to its content (complexity, explicitly, actuality and correctness) (Wang and Strong, 1996). A lack of quality on the other hand is associated to a lack of clarity, consistency, causality and intentionality which in turn leads to highly ambiguous results (March, 1994).

These considerations show, that quality measurement in knowledge transfer has to consider multiple aspects, which cannot be measured in parallel in real-life scenarios. The empirical studies outlined will therefore focus on a two stage approach, which first extracts the relevant factors under experiment conditions, and then considers relevant factors in a real-life laboratory study to investigate their interaction and their susceptibility to interventions for KM purposes.

\section{OBJECTIVES AND METHODOLOGY}

Before the empirical studies are designed and empirical methods are selected, this paper derives the artifacts required for the empirical investigation using a design-science methodology (Peffers et al., 2007). The following requirements can be specified for the empirical setting:

1. Ensure validity: The studies should satisfy both requirements for internal and external validity. While an experimental setting displays high internal validity, field studies or quasi-experiments provide better external validity (Loewenstein, 1999). Thus a sequential empirical research needs to be designed, which interlocks experimental and observational elements. 
2. Study designs as artifact: The studies must lead to insights that can be transferred among situations. For example, one situation provides a high internal validity and one situation provides a high external validity. The artifacts of two concrete research environments should demonstrate the possibility to transfer results from one research situation to another similar, more complex setting.

3. Situation analysis artifact: The artifact of situation analysis should make it possible to assess the extent to which results can be transferred from one situation to another, because knowledge transfer situations are similar.

4. Quality analysis artifact: The quality of knowledge-based process outcomes must be operationalized, so that outcomes of different situations can be compared. The artifact of quality analysis should make it possible to assess the extent to which results can be transferred from one outcome to another, because knowledge-based process outcomes are similar.

5. Influence factor analysis artifact: The influence factors must be operationalized, so that manifestations of different knowledge transfers can be compared. The artifact of influence factor analysis should make it possible to assess the extent to which results can be transferred from one knowledge transfer to another, because influence factors are similar.

\section{DESIGN}

While the first subsection addresses the operationalization of knowledge quality, the second issues situational conditions, so that experiment results can be transferred among situations. The third subsection conceptualizes knowledge transfers as they need to be examined at the studies.

\subsection{Determination of the Quality of Knowledge Artifacts}

In the early phases of PGE, models of the future product are developed. Thus knowledge artifacts are mainly models such as product concepts or process models in this phase. In accordance with contemporary approaches to measure quality of section 2.3, the assessment of model quality can be conducted using the different principles laid out by Becker et al. (1995). Variants of these principles are considered as desirable characteristics of knowledge artifacts, so that these have to be met for high quality models in knowledge-intensive processes:

1. Requirement of correctness: knowledge artifacts need to represent the expectations of the knowledge carrier and receiver at least in essential features.

2. Requirement of relevance: knowledge artifacts do not need to be complete, but it is rather necessary that the facts relevant for the purposes are represented.

3. Requirement of clarity: knowledge artifacts must be legible, understandable and as clear as possible. They should be as simple as possible and only as complicated as necessary.

4. Requirement of systematic structure: knowledge artifacts must follow a systematic structure to reduce complexity.

5. Requirement of comparability: knowledge artifacts must follow the same guidelines and rules in order to be comparable.

In accordance with ISO (2019), the scales for the five principles are operationalized with the Likert scale (Likert, 1932) describing inhowfar a certain desirable principle has been met. Insofar as several questions address a certain principle, even the percentage-oriented interpretation of the scale can be used (Table 1). Although the described requirements depend on the specific knowledge artifact, artifacts of different situations can be compared in terms of quality if they are evaluated with the same scale.

Table 1. An example operationalization of the quality of knowledge artifacts.

\begin{tabular}{lll}
\hline Scale & Description & Percentage of requirements met \\
\hline 1 & Requirements not fulfilled & $\leq 20 \%$ \\
\hline 2 & & $\leq 40 \%$ \\
\hline 3 & Requirements partly fulfilled & $\leq 60 \%$ \\
\hline 4 & & $\leq 80 \%$ \\
\hline 5 & Requirements fully fulfilled & $\leq 100 \%$ \\
\hline
\end{tabular}




\subsection{Identification of Reference Situations}

Knowledge transfers take place in different real-life situations. Since certain impediments of knowledge transfers are bound to the situational environment (Szulanski, 1996; Arrow, 1969) and further, different knowledge transfer methods will only work in specific situations (Dixon, 2000), a link between the experimental setup and real-life observation needs to be established. Here, the relevant situations need to be captured and classified. Albers et al. (2019a) identified and described 21 knowledge transfer situations during product development. From those situations, a classification schema can be derived.

First, the situations can be distinguished with regard to the number of participants involved: the archetypal knowledge-transfers internalization and externalization (Nonaka and Takeuchi, 1995) require an object and a single person, while socialization requires at least a dyad. One can thus distinguish between individual and group-oriented knowledge transfer. Latter are often accompanied by network structures between the participants which facilitate the transfer. Second, the social relationship between participants can have different degrees of power distance and imbalance. This influences the communication mode. While hierarchical transfers are clear in transporting the message, the understanding of the content allows little feedback channels. Collegial communication on the other hand occurs on the basis of an informal social structure, which take a shared understanding as a base. Communications on the basis of equal has an emergent social structure and therefore a higher complexity. It is also more prone to disruption. However, it is also more flexible in the deliberation of contested knowledge fields. Third, transfers can either be intentionally directed as in instructional settings or undirected like in team meetings, where knowledge transfer occur incidentally.

Goal orientation as the forth characteristic goes along with directionality. Situations can therefore be distinguished in how far a specific objective needs to be met during communication such as a development output, a decision or an agreement. The time frame of the knowledge transfer is a fifth criterion to classify situations. Some transfers occur on in short-term, mostly with factual knowledge. More complex knowledge transfers have either a medium or long-term orientation like re-occurring meetings or master-apprentice-relationships. Furthermore, the spacial aspects need to be considered as a sixth criterion, since the transmission of non-verbal signals provides valuable cues for the participants. Knowledge transfers in local proximity therefore provide a denser communication channel than virtual communication. The mode of the transfer process is the seventh characteristic of transfer situations, because the transfer process can be distinguished in their degree of openness. While open transfer processes do not have a specific schedule or agenda and no fixed methods, structured processes provide guidance during communication. The eighth and last characteristic is the form of the output artifact, that can be distinguished in the degree of openness, too. The outcome on the other hand can either have a fixed form, such as minutes, tests, design concepts and blueprints, or it can have an unspecific or open form, where the participants need to agree on the type of externalization beforehand.

Table 2. Morphological box of transfer situations

\begin{tabular}{|c|c|c|c|}
\hline Dimension & \multicolumn{3}{|c|}{ Characteristics } \\
\hline 1) number of participants & single & dyad & group \\
\hline 2) power distance & hierarchical & collegial & equal \\
\hline 3) directedness & \multicolumn{2}{|c|}{ directed } & undirected \\
\hline 4) goal orientation & \multirow{2}{*}{\multicolumn{2}{|c|}{\begin{tabular}{l|}
\multicolumn{2}{c}{ specific objective } \\
short-term
\end{tabular}}} & no objective \\
\hline 5) duration & & & long-term \\
\hline 6) spaciality & \multicolumn{2}{|c|}{ local } & distributed \\
\hline 7) process formalization & open & & formalized \\
\hline 8) result formalization & \multicolumn{2}{|c|}{ open } & formalized \\
\hline
\end{tabular}

Using dimensions of Table 2, each knowledge transfer activity can be described with regard to their enveloping situation and making different activities comparable.

\subsection{Influence Factors on the Quality of Knowledge Artifacts}

In accordance with the definition of knowledge transfers (see section 2.2), measuring points need to be taken along each stage of knowledge transfers (identification, transmission, application). Regarding knowledge transfers in PGE, three types of knowledge need to be issued at the experimentation: First, 
explicit knowledge or information objects refer to a well documentable form of knowledge, that can be handed among any kind of process participant easily, such as by existing product documentations, emails, wiki contributions (Nonaka and Takeuchi, 1995). Second, tacit knowledge or knowledge objects refer to a form of knowledge that is hard to document because it is knowledge-bearer-bound (Nonaka and Takeuchi, 1995). Examples refer to experiences, a feeling of essential things and intuitions. The third form of knowledge refers to physical manifestations or physical objects such as prototypes or physical models, which constitute embodied knowledge (Ignatow, 2007). In between the corner points of knowledge transfer stages as well as knowledge forms, further influence factors need to addressed as Fig. 1 shows.

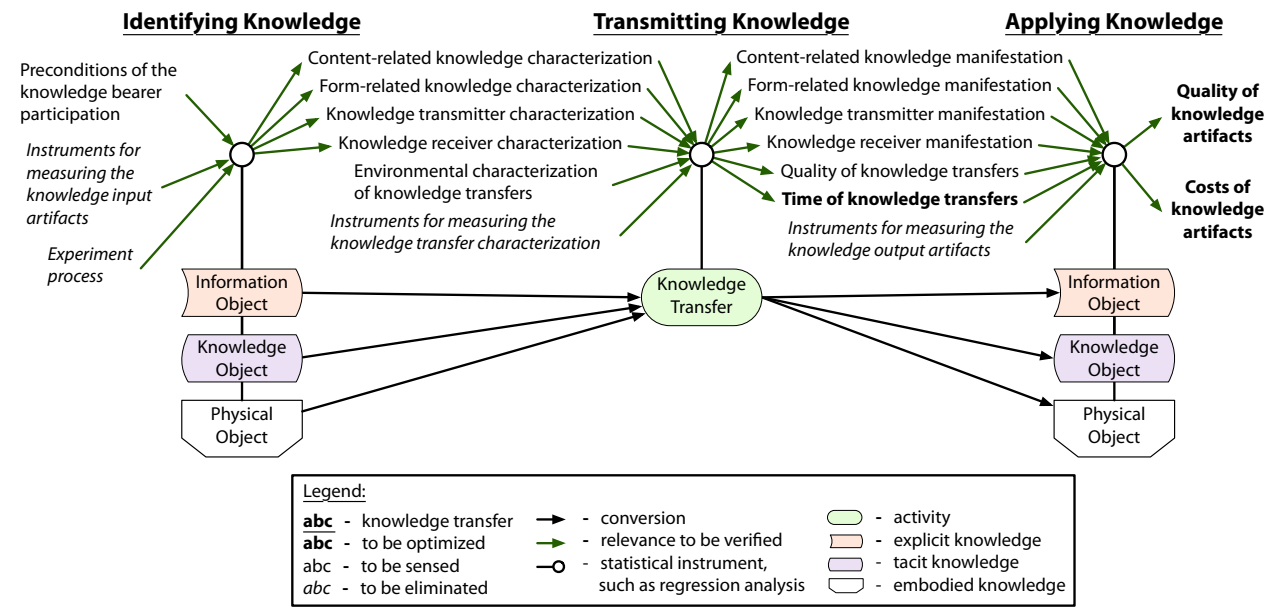

Figure 1. Conceptualization of knowledge transfers for experimentation.

In the figure, one can see relevant factors along the knowledge transfer phases. Since these are intended to be verified by the statistical instrument appliance and analysis, the experiment tool implementation will have to measure them carefully without falling to participant burden at experiment realization. They are described as follows.

Inspired by the information processing theory (Hult et al., 2004), the experimentation differentiates between knowledge carriers functioning as knowledge source and knowledge carriers that function as knowledge target. While the first transmits knowledge via different kinds of knowledge channels and the second receives knowledge, both needs to be characterized. Nevertheless if we speak about knowledge carriers as source or target, the preconditions of knowledge bearers affect the transfer. Examples refer to lacks of their motivation, their arduous relationship or their ability to perceive their environment (Szulanski, 2000).

As a coherent unity, knowledge provides a content-based dimension, which refers to the amount of interrelated information that is transferred (Boghossian, 1989), as well as a form-related dimension. The latter can be interpreted as the appearance or codification of knowledge (Zagzebski, 2017). The environmental factors of knowledge transfers have also been proven to be relevant for knowledge transfers (Szulanski, 2000). Please remark here, that the same influence factors can be identified before and after the transmitting stage. These need to be differentiated because before the transfer, knowledge objects can be characterized under the initial conditions. Because of the transmitting, they reveal as manifestation, which is the state at the applying stage. Since measurement instruments as well as the experiment process can disturb the quantitative examination of knowledge transfers, their influence needs to be minimized. As target dimensions, which have an economic meaning, we can identify the time required for knowledge transfers as well as the quality and costs of outcomes of that knowledge transfers.

\section{DEMONSTRATION}

The demonstration will show the transfer of insights from the laboratory environment to the real-life setting. For this, the research environments are concretized and artifacts created are demonstrated.

Experiments at University Potsdam: The experiment at the University of Potsdam (UP) is intended to be a labor study, that can control environmental factors (Walter et al., 2016). By selecting specific situational configurations and influencing factors, participants can be faced with knowledge-intensive tasks, 
in which they need to produce knowledge artifacts. Examples for these task are creative programming tasks such as the SQL query creation, doing business process modeling or creating a textual description of process modeling languages. Each task can be ranked by the complexity and stickiness of the knowledge involved. Furthermore the competence of the participant is assessed prior to experimentation. Tasks are performed guided by an IT system, so that different kinds of conversions and types of knowledge can be observed individually. For example, first, relevant knowledge is internalized, such as watching a video explaining how to create SQL statements or process models. Then, relevant knowledge is explicated such that a product concept manifests. For example, this refers to the creation of SQL statements, process models or descriptions about the modeling language. The overall duration of the experiment can easily be managed to not exceed two hours. The process outcomes will be evaluated in regard with the time required and their quality. Thus high internal validity can be achieved.

Live-Lab ProVIL at KIT: The live laboratory at the Karlsruhe Institute of Technology (KIT) is intended to be less bound to artificial control than the experiment of UP, so that it is situated between pure laboratory experiments and field studies (Walter et al., 2016). Since the same situational configurations and influencing factors are addressed, observations shall support a higher external validity of results. Here, ProVIL - Product development in a Virtual Idea Laboratory will provide a research environment for the evaluation of new methods, processes and tools in product development (Walter et al., 2017). In terms of the here conducted study, this is understood to be a research environment that allows both, the application of results of the laboratory study of UP and the modification of knowledge transfers to be examined in their use in a development process that is as realistic as possible. In distinction to the experiments of UP, knowledge-intensive processes are not observed individually rather than in a greater time span of real projects. Teams consist of not more than six students and the development project lasts about three month realized in parallel to regular studies. For example, the team is asked for the construction of a product enabling a VR-based learning success control, or the development of a procedure for remotely validating mechanics with the aid of $3 \mathrm{~d}$ printing technology.

\section{EVALUATION}

The evaluation intends to examine in howfar results of the one research environment, such as the experiment realized by the UP can be transferred to the other research environment such as the Live-Lab realized by the KIT.

Comparability in quality dimensions: Even though both environments produce different artifacts, they are comparable with regard to their quality as follows. In both cases, models and concepts are developed, which can be assessed with regard to the principle-based requirements laid out in section 4.1. When assessing the quality of knowledge artifacts against the same criteria, it is possible to ensure comparability between the results of the UP study and KIT study.

For example process models constructed and VR-based learning success control models developed both can be evaluated in regard with the fulfillment of the principle of clarity, if they are understandable for the target customers. They are both correct, if they address facts of a case study with a certain modeling notation without failures (process model) or learning material is questioned correctly (learning success control). They do not have to address all the facts presented rather than the relevant facts. In the case of process models, this e.g. issues relevant modeling items and in the case of learning success control, it e.g. issues essential learning objectives. Further, they both provide a systematic structure, if process model presents interwoven case studies in a simple manner and the VR-based learning success control presents tests having a systematic focus each. Lastly, the models are comparable, if models of as-is processes and to-be process, or learner performances and sample solutions can be compared.

Comparability in transfer situations: To ensure the comparability of the situations, morphological attributes to characterize situations can be considered as they were presented in section 4.2. Although both kinds of transfer situations are comparable, the differentiate in some points because of the following. While the experiments intend to focus on the examination of individual knowledge transfers, the Live-Lab tries to validate the experiment findings in practical context. With the aid of morphological dimensions worked out, the following first characterizes knowledge transfers in experiments, and then issues differences to the Live-Lab. The precise focus in experiments comes at the price of controlling the transfer situations and generating a certain amount of artificiality. The experiments concentrate on single person transfers in single experiments or dyadic transfers in team experiments. They cover the 
atomic conversions of the SECI model like internalization, externalization and extraction in the first kind of experiment as well as socialization in the second kind of experiment. Socialization is on equal grounds, as the participants do not know one another. The transfer is directed as the participants are instructed that specific checks are used to measure their success. Transfer situations are short-term with small tasks that are fulfilled. Spatiality is handled locally. Single person transfers are realized at only one work place, and dyadic transfers are realized at the same virtual conference room. The transfer process is formalized as the experiment needs to follow a predefined setup. The expected results are also formalized because the tools for the development are provided by the experiment tool. The situations in the experiments are thus defined as ideal-typical amplifying certain aspects of knowledge transfers for analytical purposes. The challenge is to transfer the findings generated from the ideal-typical situations to the realistic setting.

The Live-Lab tries to extend the findings from the experiments into more complex and ambiguous situations to constitute real-typical findings. Although both studies present specific objectives for products to be developed, transfer situations are different from those in the experiments as follows: First, the Live-Lab setting cannot control the situations as thoroughly as the experiments. While experiments can precisely modify knowledge transfers by initial configurations, the Live-Lab design allows for the manipulation during the period of three month. By deliberately designing the interventions, the experimenter can suggest certain situations to the participants. An example would be the requirement to create animations for schematic drawings, so that the stickiness is decreased (Albers et al., 2019b). However, while the team-internal communication is based on mostly equal communication, the tutor shall communicate on a collegial level, and interventions are hierarchically specified. Second, most interactions in the Live-Lab cannot be induced. It will occur endogenous due to group dynamics. Especially during creative phases, there will be a mix of different ideal-typical transfers. As most of the tasks are creative and problem solving, these transfer situations are expected to be undirected, in an open process with open result's format. In order to enable the comparability, each intervention therefore needs to be classified with regard to the transfer situation it tries to induce. Furthermore it needs to be observed if the expected situation has actually occurred during the team session. Third, transfer situations can furthermore be short-term to medium-term in the Live-Lab setting, as the teams have reoccurring meetings and need to solve problems within and between the meetings. In distinction to the local knowledge transfers of the experiments, the Live-Lab provides knowledge transfers locally in the same meeting rooms as well as distributedly when working individually. The interactions therefore need to be observed and classified with regard to their nature. The schema in Table 2 provides guidance and ensures a coherent, comparable situation description and association between situation and resulting artifact.

Comparability in influence dimensions: To ensure the comparability of the knowledge transfers in the situations described, influence factors to characterize the effect on knowledge-based process outcomes have been identified. These were presented in section 4.3. Based on their concrete operationalization, quantitative effects of knowledge transfers can be found again if the following holds:

First, knowledge transfers at the experiments and the Live-Lab comprise the same measure points, if both the transfer at the creation of process models and the creation of an learning success control concept comprise the identification, transmission and application of knowledge. Second, knowledge transfers clamp the same forms of knowledge. For example, the process model creators and the VRproduct creators both transfer their tacit knowledge to an explicit form of knowledge, which is in the first case the process model and in the second case is the written concept. Third, knowledge carriers as source and target need to provide the same preconditions and they need to use the same knowledge channels. For example, all the knowledge carriers need to be motivated and to speak in their mother tongue. Fourth, the appearance and codification of knowledge need to be similar. For example, the amount of interrelated information is comparable if the process models and VR-based learning success control concepts satisfy the same amount of requirements. Their form-related dimension is comparable if both process outcomes refer to a schematic drawing. Fifth, knowledge transfers at the experiments and the Live-Lab are realized under the same environmental conditions. For examples, one can find both situations the soundscape of an open-plan office and the test persons have a realistic time pressure. Sixth, the relevant outcome dimensions time-quality-costs are measured in the same scale. For example, both situations determine the costs of knowledge transfer outcomes in Euro. 


\section{CONCLUSION}

Critical appraisal: In regard with the research question ("How can a practical experimental environment be designed that allows the quantitative investigation of factors influencing the quality of knowledge transfer outcomes?") one can say that the artifacts of an 1) situation analysis, 2) quality analysis and 3) influence factor analysis have been designed and applied to two different kinds of empirical study forms. The artifacts have enabled the comparison of the different knowledge transfer situations, so that one can evaluate if it is possible to transfer the results of the experiments and the Live-Lab. On the basis of their demonstration, several extra conditions have been identified, which need to be considered at the study realizations, so that findings of both studies can be generalized correctly.

Impact on product development: If quantitative effect of factors influencing the knowledge transfer quality has been determined and interventions improving knowledge transfers have been empirically verified, findings will lead to competitive advantages in real-life business contexts. Assumed the complexity of an engineering task is to high in regard with the engineering competence, equivalent to time improvement of Grum et al. (2019), output quality might be improved if a) components to be constructed are divided up into two sub-components and are less complex, b) component-specific education material is provided and competence is raised, and c) stickiness is reduced by the use of common terms.

Limitations and outlook: Due to the absence of concrete tasks at the situations of the experiments and the Live-Lab, the application of artifacts designed only has been demonstrated exemplary. So far, the evaluation has been realized on basis of argumentation, which lacks in validity of the artifacts. This might be addressed by their validation in expert workshops, surveys, pretests, and the realization of both of the research environments presented. For this, concrete experiment tools and interventions will be designed and implemented. The selection of interventions will base on the findings of experiments.

\section{REFERENCES}

Albers, A., Bursac, N. and Wintergerst, E. (2015), "Produktgenerationsentwicklung - Bedeutung und Herausforderungen aus einer entwicklungsmethodischen Perspektive", in: Stuttgarter Symposium für Produktentwicklung, Vol. 2015.

Albers, A., Gronau, N., Rapp, A., Grum, M., Zaiser, A. and Weber, E. (2019a), Influencing factors and methods for knowledge transfer situations in Product Generation Engineering based on the SECI model, chapter Knowledge Transfer Velocity Model Implementation, Empirical Studies of Business Informatics, GITO, pp. $8-22$.

Albers, A., Lüdcke, R., Bursac, N. and Will, J. (2014), "Process analysis and optimization by targeted application of knowledge management - a case study in the early stages of product development", in: Methoden in der Produktentwicklung: Kopplung von Strategien und Werkzeugen im Produktentwicklungsprozess : 12. Gemeinsames Kolloquium Konstruktionstechnik Bayreuth, 16. - 17. Oktober 2014. Ed.: F. Rieg, Lehrstuhl für Konstruktionslehre und CAD, Universität Bayreuth, pp. 33-42.

Albers, A., Rapp, A. and Grum, M. (2019b), Knowledge Transfer Speed Optimizations in Product Development Contexts, chapter Knowledge Transfer Velocity Model Implementation, Empirical Studies of Business Informatics, GITO, pp. $93-104$.

Arrow, K.J. (1969), "Classificatory notes on the production and transmission of technological knowledge", The American Economic Review, Vol. 59 No. 2, pp. 29-35.

ASQ (2020), Letter Q - Quality Glossary of Terms, Acronyms and Definitions with Letter Q, Quality glossary, American Society for Quality, Available at: https://asq.org/quality-resources/qualityglossary/q.

Becker, J., Rosemann, M. and Schütte, R. (1995), "Grundsätze ordnungsmäßiger Modellierung", Wirtschaftsinformatik, Vol. 37 No. 5, pp. 435-445.

Boghossian, P.A. (1989), “Content and self-knowledge", Philosophical Topics, Vol. 17 No. 1, pp. 5-26.

Connelly, C., Ford, D., Turel, O., Gallupe, B. and Zweig, D. (2014), “'i'm busy (and competitive)!' antecedents of knowledge sharing under pressure", Knowledge Management Research \& Practice, Vol. 12 No. 1, pp. 74-85.

Crosby, P.B. (1979), Quality is free: The art of making quality certain, Vol. 94, McGraw-hill, New York.

Dixon, N.M. (2000), Common knowledge: How companies thrive by sharing what they know, Harvard Business School Press.

Drucker, P. (1994), Post-capitalist Society, Butterworth-Heinemann.

Eckert, C., Alink, T., Albers, A. et al. (2010), "Issue driven analysis of an existing product at different levels of abstraction", in: DS 60: Proceedings of DESIGN 2010, the 11th International Design Conference, Dubrovnik, Croatia, pp. 673-682. 
Gronau, N. (2019), Knowledge Modeling and Description Language 3.0 - Eine Einführung, Vol. 1, GITO mbH Verlag Berlin.

Gronau, N. and Grum, M. (2019), Knowledge Transfer Speed Optimizations in Product Development Contexts, chapter Towards a prediction of time consumption during knowledge transfer, Empirical Studies of Business Informatics, GITO, pp. $25-69$.

Grum, M. and Gronau, N. (2018), “A visionary way to novel process optimizations - the marriage of the process domain and deep neuronal networks", in: B. Shishkov (Editor), Business Modeling and Software Design, Springer International Publishing, Cham, pp. 1-24.

Grum, M., Rapp, S., Gronau, N. and Albers, A. (2019), “Accelerating knowledge - the speed optimization of knowledge transfers", in: B. Shishkov (Editor), Business Modeling and Software Design, Springer International Publishing, Cham, pp. 95-113.

Hult, G.T.M., Ketchen, D.J. and Slater, S.F. (2004), "Information processing, knowledge development, and strategic supply chain performance", Academy of Management Journal, Vol. 47 No. 2, pp. 241-253.

Ignatow, G. (2007), "Theories of embodied knowledge: New directions for cultural and cognitive sociology?", Journal for the Theory of Social Behaviour, Vol. 37 No. 2, pp. 115-135.

ISO, I. (2019), "Iec 25030 software and system engineering-software product quality requirements and evaluation (square)-quality requirements", International Organization for Standarization.

Likert, R. (1932), “A technique for the measurement of attitudes", Archives of Psychology, Vol. 22 No. 140, p. 55.

Lindemann, U., Lorenz, M. et al. (2008), "Uncertainty handling in integrated product development", in: DS 48: Proceedings DESIGN 2008, the 10th International Design Conference, Dubrovnik, Croatia, pp. 175-182.

Loewenstein, G. (1999), "Experimental economics from the vantage-point of behavioural economics", The Economic Journal, Vol. 109 No. 453, pp. F25-F34.

March, J. (1994), A Primer on Decision-Making, Free Press, New York, NY.

Nonaka, I. and Takeuchi, H. (1995), The knowledge-creating company: How Japanese companies create the dynamics of innovation, Oxford university press.

Peffers, K., Tuunanen, T., Rothenberger, M.A. and Chatterjee, S. (2007), “A design science research methodology for information systems research", Journal of management information systems, Vol. 24 No. 3, pp. 45-77.

Rhodes, J., Hung, R., Lok, P., Lien, B.Y.H. and Wu, C.M. (2008), "Factors influencing organizational knowledge transfer: implication for corporate performance", Journal of knowledge management, Vol. 12 No. 3, pp. 84-100.

Sommerlatte, T., Beyer, G. and Seidel, G. (2006), Innovationskultur und Ideenmanagement, Symposion Publishing GmbH.

Szulanski, G. (1996), "Exploring internal stickiness: Impediments to the transfer of best practice within the firm", Strategic Management Journal, Vol. 17 No. S2, pp. 27-43.

Szulanski, G. (2000), "The process of knowledge transfer: A diachronic analysis of stickiness", Organizational Behavior and Human Decision Processes, Vol. 82 No. 1, pp. 9-27.

Walter, B., Albers, A., Benesch, G. and Bursac, B. (2017), "ProVIL - Produktentwicklung im virtuellen Ideenlabor: Anwendungs- und Implementierungsmodell eines Live-Labs", in: H. Binz (Editor), 4. Stuttgarter Symposium für Produktentwicklung 2017, Fraunhofer Verlag, Stuttgart, Deutschland.

Walter, B., Albers, A., Haupt, F., Bursac, N. et al. (2016), "Produktentwicklung im virtuellen Ideenlabor Konzipierung und Implementierung eines Live-Lab", in: DFX 2016: Proceedings of the 27th Symposium Design for X, 5-6 October 2016, Jesteburg, Germany, pp. 283-295.

Wang, R.Y. and Strong, D.M. (1996), "Beyond accuracy: What data quality means to data consumers", Journal of management information systems, Vol. 12 No. 4, pp. 5-33.

Zagzebski, L. (2017), What is Knowledge?, chapter 3, John Wiley and Sons, Ltd, pp. 92-116. 